\title{
APRON: A Cellular Processor Array Simulation and Hardware Design Tool
}

\author{
David R. W. Barr and Piotr Dudek \\ School of Electrical and Electronic Engineering, The University of Manchester, P. O. Box 88, Manchester M60 1QD, UK \\ Correspondence should be addressed to David R. W. Barr, d.barr@postgrad.manchester.ac.uk
}

Received 12 September 2008; Accepted 21 March 2009

Recommended by David Lopez Vilarino

\begin{abstract}
We present a software environment for the efficient simulation of cellular processor arrays (CPAs). This software (APRON) is used to explore algorithms that are designed for massively parallel fine-grained processor arrays, topographic multilayer neural networks, vision chips with SIMD processor arrays, and related architectures. The software uses a highly optimised core combined with a flexible compiler to provide the user with tools for the design of new processor array hardware architectures and the emulation of existing devices. We present performance benchmarks for the software processor array implemented on standard commodity microprocessors. APRON can be configured to use additional processing hardware if necessary and can be used as a complete graphical user interface and development environment for new or existing CPA systems, allowing more users to develop algorithms for CPA systems.
\end{abstract}

Copyright ( 2009 D. R. W. Barr and P. Dudek. This is an open access article distributed under the Creative Commons Attribution License, which permits unrestricted use, distribution, and reproduction in any medium, provided the original work is properly cited.

\section{Introduction}

Massively parallel processing systems have been the topic of high-performance computing research and design for many years $[1-4]$, but in recent years single-chip implementations of such systems are becoming a reality. Cellular processor arrays (CPAs) such as the ones presented in [510] implement data processing at a fine-grain level of parallelism. Unlike sequential and coarse-grain parallel processing systems, such as multicore processors [11-13], which have large and complex instruction sets, CPAs are comprised of simpler processors, with a reduced instruction set. Typically they are used to perform lower-level data-parallel algorithms and operate in the Single Instruction Multiple Data (SIMD) mode, that is, every processor executes the same instruction but operates on their own local memories. The processors require much less silicon area due to their simplicity (compared to standard microprocessors) which means high numbers of them can be placed on a device, usually arranged in a $2 \mathrm{D}$ grid (Figure 1). The massively parallel nature of the device and spatial positioning of the processors give performance and efficiency advantages, in particular in data-intensive algorithms which require a high memory bandwidth, as each processor is able to access its own data and exchange data with its neighbours. A nonparallel system would have to perform many instructions, including memory sequential accesses to accomplish the same task. The low power consumption of a CPA, significantly lower than that of an equivalently performing sequential system (such as a desktop PC or workstation), is an appealing property for designers of low power embedded systems.

The highly parallel nature of CPAs has increased appeal to a variety of fields, where many simple computations must be performed on data arrays. The applications include embedded vision systems [6], high-speed image processing $[5,8]$, cellular nonlinear networks (CNNs) $[7,14]$, and computational neuroscience models [15-17]. These applications often require simple numerical operations and neighbour communication but use operations that are applied homogeneously to large data-sets, at high speeds. The fine-grain nature of many CPAs means that thousands of local memories can be operated on simultaneously, and the simplicity of the processors allows for fast execution of instructions.

The design of CPAs presents a set of challenges. It requires difficult trade-offs and design decisions, such as accuracy or speed, analogue or digital, and memory or array size. The drive to achieve a high density of processors in 


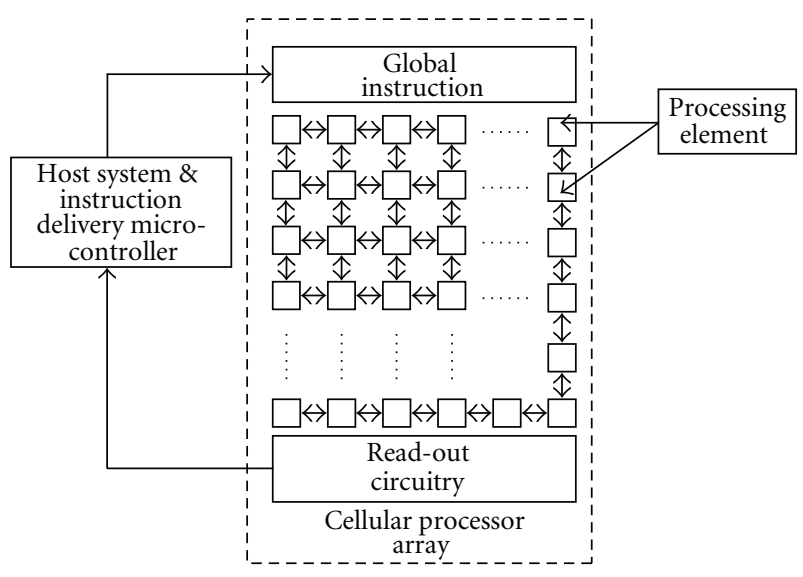

FIgURE 1: An overview of a simple cellular processor array system.

a single chip often makes memory a limited resource and reduces their computational ability. Also, the specialised nature of the hardware often makes it less accessible to potential application developers, while typically used short word lengths or analogue storage techniques limit their accuracy. Further to this, many CPAs require additional interfaces and control systems which are specifically built for the device, introducing additional design costs and possibly restricting the performance of the overall CPA system.

This paper is an extended version of work presented in [18] and presents APRON (Array Processing enviRONment), a high-speed, flexible, and virtual CPA implemented entirely in software. The software fulfils two objectives: firstly to provide an extensible, customisable implementation of a general purpose array processing engine, which is a basis for the design and "virtual prototyping" of new CPAs; secondly to provide a high-speed emulation of any CPA-based device, allowing the user to design pixel-parallel image processing algorithms and explore multilayer neural networks, cellular automata, and other phenomena related to CPAs, with a variety of tools for data analysis, performance evaluation, and algorithm development. APRON software is written to take advantage of the high-speeds of modern desktop computer systems to provide optimised array processing performance. To this end it is not intended to replace low-power embedded devices but serves as a fast, accurate substitute in environments where custom hardware is not available. APRON has been designed to provide user-friendly tools and achieve the maximum computational performance, with minimal overheads, without the need for custom low-level implementations.

This paper is organised as follows: Section 2 presents the APRON simulation core and the integrated development environment (IDE); Section 3 presents the potential applications/research fields where APRON can be used effectively and how the system can emulate several types of CPA device simultaneously; Section 4 highlights the flexible architecture of the APRON compiler and illustrates how it can be modified to emulate or prototype hardware devices; Section 5 presents a performance analysis and benchmarks of the APRON system. Throughout the paper, performance figures are presented, which are summarised in the conclusion.

\section{APRON System Overview}

2.1. The Simulation Core. The APRON simulation core is a platform-independent module that can be used in many applications for fast array processing in software. The core provides a virtual array processor, operating on three data types with a flexible instruction set and a virtual microcontroller. The microcontroller controls the program counter which permits nonlinear sequencing of algorithm instructions. APRON by default uses 32-bit single precision floating point numeric representations in all operations but it is possible to change this if necessary to lower bit-size integer types. The data types are registers, kernels, and linkmaps.

Registers are two-dimensional arrays of numeric elements called cells, which are 16-byte aligned in memory contiguously, to facilitate cache/page management and to take advantage of the extended x86 intrinsic architectures: SSE (Streaming SIMD Extensions) and SSE2 [19]. These architectures provide an SIMD vector processor in the silicon fabric of the CPU. This vector processor can process four single-precision floats in a minimal number of clock cycles, providing a calculation throughput much faster than that of the standard floating point unit (FPU). SSE also provides cache management facilities, meaning that registers can be pre-cached close to the processor, and even read/written straight from/to the main system memory, without polluting the cache if necessary. APRON registers adopt one of two forms. A data register contains numeric data, and a mask register contains binary data, which is used to enable and disable the write privileges to a data register, in effect permitting regional register operations (where global register operations are default). For many operations, the target register can also be a source register due to the sequencing of instructions sent to the CPU (e.g., giving $x=x+1$ functionality). Figure 2 shows how data is sequenced, sent to the vector processor, and combined with a mask register to emulate the effect of disabling a processing element's "enabled flag", providing local autonomy. The contents of the target register should only be updated if the mask register's corresponding bit is set. This requires additional logic when working with a vector of four elements, of which only some of the corresponding mask register bits are set, to form a combined result vector, which is written to the target register. The mask register contains a 32-bit floating point $\mathrm{NaN}$ (0xFFFFFFFF), for each processing element that is enabled, and a $0(0 \times 00000000)$ for those that are disabled. Whilst at first this may seem like a gross overuse of memory, having all the bits set to 1 is useful for rapid logic functions; in this case a logic OR is computed between the data register's vector and the corresponding mask vector. This is an efficient way of implementing branching in the vector processor.

A kernel is an $N \times N$ matrix used in two-dimensional convolution/filtering operations and is applied to a register. Kernels come in two forms. A standard kernel is used in typical greyscale image filtering operations (convolutions), such as Gaussian blur and gradient-detect. A template kernel is a binary kernel used for binary pattern matching, comprising of hits, misses, and do not cares. A logic OR operation is performed within the domain of the kernel 


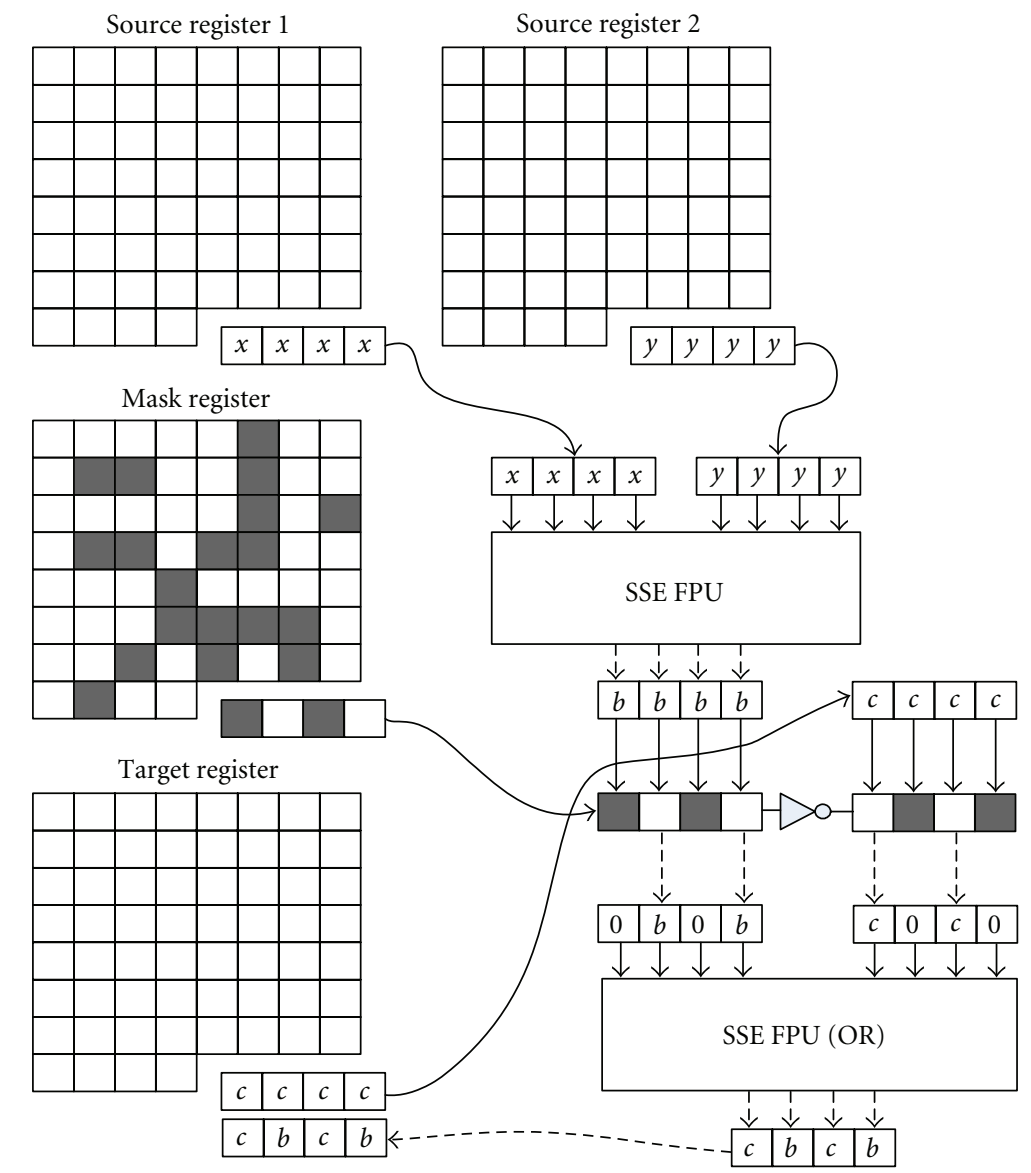

FIGURE 2: Disabling processor elements is achieved by restricting writes to the register memory by using the mask register in a sequence of logic operations. Dashed lines are memory writes, and the others are reads.

and the register, and the binary result is placed in a result register. Unfortunately, SSE2 does not offer a simple way of executing the kernel operation quickly, due to a lack of efficient sideways communication within the vector processor (however SSE3 does support this and is becoming standard on modern processors). Instead, APRON ensures all data required is in the highest level of cache, as close to the processor as possible, reducing cache misses (and eliminating paging), and the OS is stopped from swapping out the APRON process for the duration of the operation. The code is written in such a way as to be optimised greatly by the $\mathrm{C}++$ compiler. The disassembly shows that 95 assembly level instructions are required for a fully filtered pixel using a $3 \times 3$ matrix.

Linkmaps are a structured list of all the connections from a single cell. APRON supports local neighbour connectivity by default, so registers can be shifted and rotated in the 4 standard compass directions, but by having no hardware connectivity limitations, it is possible to have any cell connected to any number of cells anywhere within the register. Linkmaps exist for each cell in the register, allowing unlimited connectivity possibilities. Each link is also accompanied by a multiplicative coefficient which is analogous to a synaptic weight (See Figure 3 ). When a linkmap is used, each source register value is multiplied by each of its connections defined by the linkmap. Each target register value is the result of an accumulation function, which is either a summation or maximisation of all the multiplicative connections that link to that value's location. The APRON IDE allows the algorithmic generation of linkmaps through a built-in Python interpreter and a set of parametric maps (registers pre-filled with data), which means the connectivity output from a register's cell can vary in a spatially controlled way. Linkmaps can become large, for a fully specified linkmap of a register size $N \times N$; $\mathrm{N}^{4}$ connections are required. Often this is not the case, so connections below a specified value are assumed to be negligible and are omitted from the linkmap list.

A variation of the linkmap is the parametric linkmap, in which the connectivity is described parametrically; for example, all of the connections are based on the same Gaussian equation, but different coefficients can change the shape of that equation. Currently, this is computationally time consuming and is the subject of ongoing research, as the potential of using additional special-purpose hardware to perform this task could greatly reduce the memory bandwidth requirements of a fully connected linkmap. APRON is a vehicle for testing various connectivity acceleration strategies, whilst keeping the model structure intact, permitting fair benchmark comparisons between each technique. 
Source register

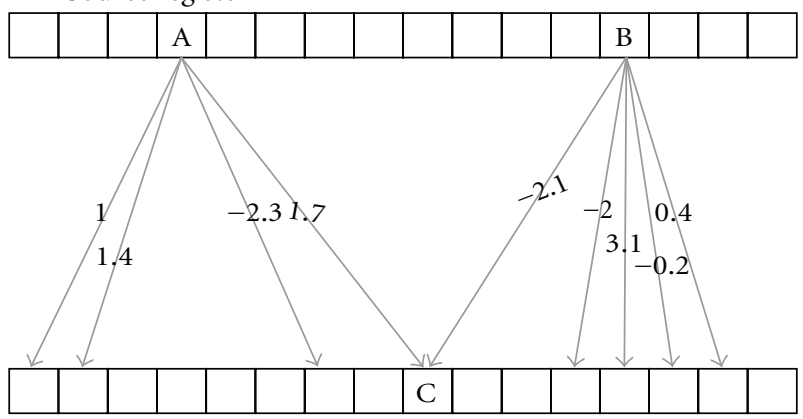

Target register

Figure 3: Linkmaps allow multiplicative connections between the processing cells. Each cell can be connected to any other cell. Here, different linkmaps are shown for cells $\mathrm{A}$ and $\mathrm{B}$, where $\mathrm{C}=$ $1.7 \mathrm{~A}-2.1 \mathrm{~B}$.

Linkmaps allow modelling of CPAs with elaborate interprocessor connectivity patterns. However, their main use is for implementing multilayer topographic neural networks and other models in the field of computational neuroscience. The use of APRON in this context will be elaborated in our future publications.

The simulation core has been designed to be extensible, so if necessary additional hardware (such as FPGAs or existing processor arrays) can be used to increase the computation speed. Currently the core supports 63 operations/instructions, including mathematical operators, register translation, filtering, linkmap training, and external data transfer. A simple plug-in interface permits users to build their own modules, which can be accessed at runtime during the simulation for transferring/computing register and other data. In effect, this allows any form of operation to be performed. Being a "virtual processor array" many techniques which are difficult to implement in hardware are relatively simple in APRON, an example being subwindowing and scaling of registers, where isolated regions of registers can be transferred to different locations in other registers.

\subsection{Flexible Simulation Compiler and Simulation Environ-} ment. To accompany the simulation core, a flexible compiler has been developed that allows the user to develop algorithms, systems, and model devices in an intuitive way. Consequently, a special purpose scripting language called "APRON-Script" has been developed which is easy to use and serves as the underlying description mechanism for all simulations, prototypes, and algorithms. All APRONScript code can be checked for syntax errors and validated automatically. The scripting language does not require variable declaration and operates on an assumption that one line of code corresponds to one hardware instruction. This makes looking for performance bottlenecks and optimisation easier. Multiple source-code files can be combined into a single project, which is subsequently compiled into an object code for the simulator. This promotes source-code reuse and eases the amalgamation and distribution of projects.
The user can define environmental constraints such as the number and dimensions of registers, constants, and macros for a specific device they are emulating, which can be given to other users who wish to develop algorithms for that platform. By using a stand-alone compiler, a variety of popular source editing tools can be used, and user-friendly attributes such as on-line documentation and syntax highlighting are available, promoting more rapid development.

The simulator provides tools for executing and analysing algorithms. All registers are visually viewable as 2D images and can be numerically inspected. The user can step through code or enter a "run" mode, which executes the algorithms as fast as possible. For long simulations on slower computers, a low-priority run mode is provided. The simulator also provides dynamic input/output with some included plugins. Currently APRON provides a module to read and write data to files, a module to interface with an imaging device (e.g., a webcam) for capturing real-time visual input and a module to communicate with other APRON simulations over a network, providing a convenient way of implementing large-scale simulations. A module could also be an interface to external hardware devices, such as robotic arms, pan, tilt chassis, and so forth, in effect enabling APRON to be a development environment for intelligent systems or autonomous agents, with sensor input, efficient image and neural processing, and actuator output. An APRON script algorithm can also send interrupts to the simulator to perform tasks, such as update the display, reposition windows, display performance information, issue breakpoints, and display run-time error information. Simulations can be terminated at any time. Each instruction is timed and displayed, for identifying performance bottlenecks.

\section{Applications}

3.1. Generic Processor Array. APRON has been optimised for speed and accuracy. Using just the default APRON-Script instructions, it is possible to implement a wide range of array processing algorithms. Essentially, APRON is still a serial processing system, and so the smaller the registers, the better the performance. The benchmarks in Figure 7 show that for simple numeric operations, global register $(128 \times$ 128) operations occur in several microseconds, meaning tens of thousands of register operations can be performed per second. This would imply APRON could potentially be used in both practical and research applications. Algorithms are written in default APRON-Script, which in this scenario can be considered a high-level language. The optimised simulation core allows APRON to outperform popular alternatives such as MATLAB and regular (naïve) $\mathrm{C} / \mathrm{C}++$ implementations.

3.2. Vision Systems. The APRON simulator allows registers to be filled with data acquired from an imaging device (e.g., a webcam). Therefore live image data can be used during simulation. When operating in "run" mode, many algorithms execute fast enough to be interactive and responsive to the visual input. Taking Sobel Edge detection as an 


$$
\begin{gathered}
G_{x}=\left[\begin{array}{rrr}
+1 & +2 & +1 \\
0 & 0 & 0 \\
-1 & -2 & -1
\end{array}\right] \times A \quad G_{y}=\left[\begin{array}{rrr}
+1 & 0 & -1 \\
+2 & 0 & -2 \\
+1 & 0 & -1
\end{array}\right] \times A \\
G=\sqrt{G_{x}^{2}+G_{y}^{2}}
\end{gathered}
$$

FIgURe 4: The Sobel Operator equations, used in APRON benchmarks, where $G$ is the result of a filtered pixel at location $(x, y)$, and $A$ is the unfiltered image.

example (see Figure 4), APRON can complete this algorithm for a $128 \times 128$ array in $0.5 \mathrm{~ms}$. Imaging devices vary in capturing/transfer time, but webcams typically take between 10 and $100 \mathrm{~ms}$ to both capture and transfer an image into APRON. Therefore, a complete capture-process cycle can take $10.5 \mathrm{~ms}$, giving a frame-rate of $95 \mathrm{fps}$. Higher quality cameras may bring this down to $2 \mathrm{~ms}$, giving $400 \mathrm{fps}$. Vision systems implemented in the APRON virtual array processor can be considered similar to pixel-parallel vision chip systems [20]. For $3 \times 3$ filtering of a $128 \times 128$ array, APRON has a throughput of $81 \mathrm{MP} / \mathrm{s}$ (Mega-Pixels/Second) and for simple operations (add/multiply) $958 \mathrm{MP} / \mathrm{s}$; for $512 \times 512$ this is $51 \mathrm{MP} / \mathrm{s}$ and $244 \mathrm{MP} / \mathrm{s}$, respectively. For comparison, in [21], a $3 \times 3$ filter on a $3.0 \mathrm{Ghz}$ Pentium 4 processor gives $9.7 \mathrm{MP} / \mathrm{s}$; a Xilinx Virtex-II Pro, $202 \mathrm{MP} / \mathrm{s}$; an $n$ Vidia 6800 Ultra GPU, $278 \mathrm{MP} / \mathrm{s}$. These results do not take into consideration any other processing or communication requirements, unlike APRON. It may often be the case that actually transferring the data to/from these devices has a substantially negative effect on the performance of the system as a whole.

3.3. Cellular Nonlinear Networks. APRON is easily configured to behave as a Cellular Nonlinear Network (CNN), for example, implementing the classical Chua model [22]. As with the Vision System description previously, an imaging device can be used as input. The performance of the model varies with the array size and the type of algorithm executing (timing and iteration requirements vary). For example, APRON can perform the "greyscale feedback convolution" task in $180 \mu \mathrm{s}$, which could be compared with results presented in [7].

3.4. Topographic Neural Network Models. These models often represent abstractions of known neuroanatomy, where neurons are grouped into layers and can have complex interlayer connectivity, such as those described in [15-17]. Usually special purpose software is developed to simulate such models as in [23], but APRON can be configured to perform these simulations taking advantage of its optimised routines. The high numerical precision and specific instruction set of APRON enables the implementation of many types of neural network models, using array registers in a way similar to that in [24]. Unlike hardware processor arrays, APRON has fewer limitations on resources used, supporting over 32000 registers (of a maximum $2048 \times 2048$ elements). Neurons are arranged into homogeneous layers, the size of a register; however, the neuron parameters need not be homogeneous. For example, a layer of Izhikevich [25] neurons can be implemented using 2 registers to store the states, 1 register to store the spike output, and several temporary registers for calculating the state update. Network connectivity is provided through the use of linkmaps described previously. On a $1.8 \mathrm{GHz}$ Intel Core2 Duo processor, an Izhikevich neuron takes approximately $21 \mathrm{~ns}$ to update, potentially allowing over 45000 neurons to be updated in real time with a $1 \mathrm{~ms}$ time step. Gaussian receptive fields can be implemented either through linkmaps or by using a Gaussian blurring filter on a register filled with the output of the layer [24]. A single $3 \times 3$ blurring filter applied to a $128 \times 128$ register takes approximately $170 \mu$ s.

Combining the "virtual" vision chip and processor array results in a powerful modelling tool suitable for use in retinal and visual-cortical simulations, often in a more controllable environment than that delivered by hardwareoriented systems. This flexibility allows the developer to create entire "system-on-virtual-chip" applications that may execute at speeds suitable for practical application use.

\section{Hardware Prototyping/Emulation}

One definite benefit a software CPA has over hardware is the relatively unlimited possibilities for processor element complexity, communication strategy, and memory resources. APRON provides many optimised operations (here considered low level) which can be combined to form higher-level more complex macros. This ensures that the CPA designer is using optimised code to implement the functionality of a cell's processing element. APRON provides at the very least the ability to read and write to each cell individually, which is sufficient to implement the most complex and diverse operations.

\subsection{Customizing APRON for Device Emulation. Figure 5} shows the data flow through the APRON compiler and simulator. The APRON compiler has three levels of abstraction. The highest is the scripting language itself, which is human readable, and consists of a combination of APRON instructions and macros. A macro is similar to a function, in that it encapsulates many APRON instructions into what appears to be a single instruction, except it becomes explicitly unrolled into the instruction sequence rather than placed onto the stack when called. Macros have global bodies, that is, they can access any resource, but have local labels and variables as well, to maintain the encapsulation. For example, in the scenario where the same macro is called twice, it is important not to have one macro jump to instructions in the other macro. Macros are defined in external "ruleset" files (akin to "header files" programmed in APRON-Script syntax), which also define resource name changing, constant declaration, operator overloading, and some environmental properties such as Register dimensions. The simulator provides a facility to debug the macros verbosely, in order to find errors in the ruleset.

Using macros, a cellular processor array designer could specify the complete instruction set of a hardware device, knowing the APRON instructions will deliver repeatable results. This is useful for both the emulation of existing 


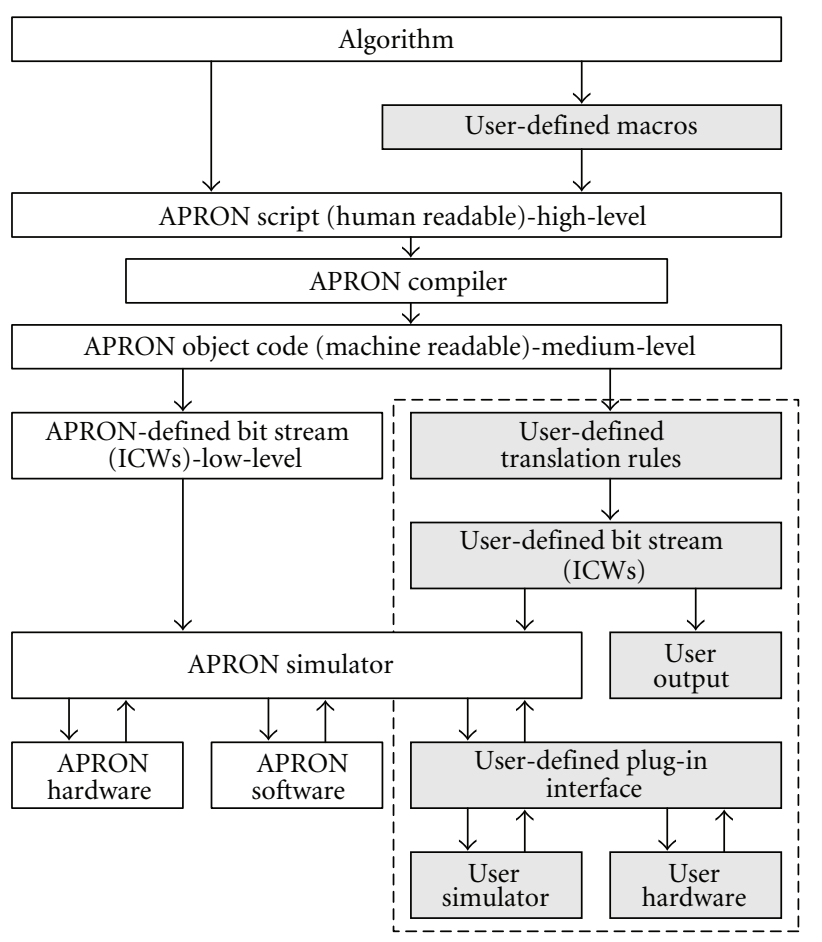

Figure 5: APRON Compilation/System Diagram. The shaded regions show user configurable components that customize APRON. The boxed region indicates components that need to be programmed and imported as a dynamic library.

hardware and the prototyping of new hardware. The designer could build several rulesets, describing the hardware at different levels of abstraction, from an accurate, slower model of the analogue current or bit transfers/operations in the individual cells to a faster, abstract behavioural model of the device. In particular, when designing analogue processor arrays, it is possible to model low-level circuit and device behaviour including spatial and temporal noise, by emulating such components. Although APRON does not simulate these effects intrinsically (i.e., there is no dedicated support for individual device characteristics), they can be sufficiently modelled using the constructs APRON provides to create accurate real-world behaviours, allowing algorithm designers to take phenomena such as nonlinearities, error propagation, and device mismatch into account [26]. The APRON IDE already provides the tools for the development and analysis of algorithms and a fast interactive simulation tool; therefore, the designer does not have to create any software himself. The "ruleset" can be distributed to any user that wishes to develop algorithms for the particular CPA. This approach has been applied successfully to the ASPA vision chip [5], and APRON is used for algorithm development for that device. APRON will also be used for the development of SCAMP devices [6].

The middle layer of abstraction is APRON object code, which is a machine readable structure defining each instruction in the APRON-Script algorithm, after all necessary preprocessing (such as macro unrolling) has been applied. This structure breaks down the script instruction into a function name and a variety of parameters. The object code by default is then turned into a binary Instruction Code Word (ICW) stream. A single object-code instruction can consist of many ICWs. The ICW stream is the lowest level of abstraction in the APRON system. This stream is delivered to the APRON Core for execution

The module responsible for the translation from object code to an ICW stream is hot swappable, with a defined interface, meaning it is possible for designers to create their own ICW streams. This has the following several benefits.

(1) The user can use the APRON environment as a code editor/compiler for a custom CPA.

(2) The ICWs can be delivered to other applications, and even hardware devices.

(3) The ICWs can be delivered to a custom simulation core, by passing the APRON Core entirely, providing a highly customised simulation, but utilising the development environment and tools APRON provides.

(4) It is similar to 3 but actually uses a hardware device and APRON as a software interface or graphical user interface to the hardware.

4.2. Modelling ASPA in APRON. The ASPA vision chip [5] is a digital cellular processor array, which contains a bit-serial/bit-parallel synchronous data path as well as asynchronous wave-propagation circuitry. This allows a wave edge to be propagated across the CPA in a single instruction, very quickly. Therefore it has many applications in more complex image processing tasks, such as skeletonisation and object identifying. The traditional approaches to building a complete CPA system involve building the device, then building an accompanying interface system, and then designing some compiler and simulation tools. APRON is being used for the latter, with a little customisation.

Firstly, the APSA instruction set was completely modelled in detail in APRON, by defining a ruleset, which is a set of macros built entirely in APRON-Script, one corresponding to each ASPA instruction. This limits the algorithm developer to only using these macros and therefore only using valid ASPA instructions. The resulting "ASPAScript" can be compiled in two ways. The first is to generate the actual hardware ICWs and store them in a file, which can subsequently be used to program the ASPA device. (The hardware ICWs may differ from those that are created for the simulator and can be defined algorithmically as part of the macro definitions.) The second is to unroll the ASPA-Script macros back into APRON-Script and run this code in the simulator. The process of simulating when using a ruleset is invisible to the algorithm developer, as the simulator presents the ASPA-Script for debugging and analysis. The detail of the ruleset can be targeted for different types of simulation. The most detailed simulation involves modelling the bitlevel transfers between the registers in ASPA, providing good debugging information at the expense of performance. A higher-level simulation may assume the user is using 8-bit integers and model the device at that level, providing an adequate simulation of the device at high speed. 


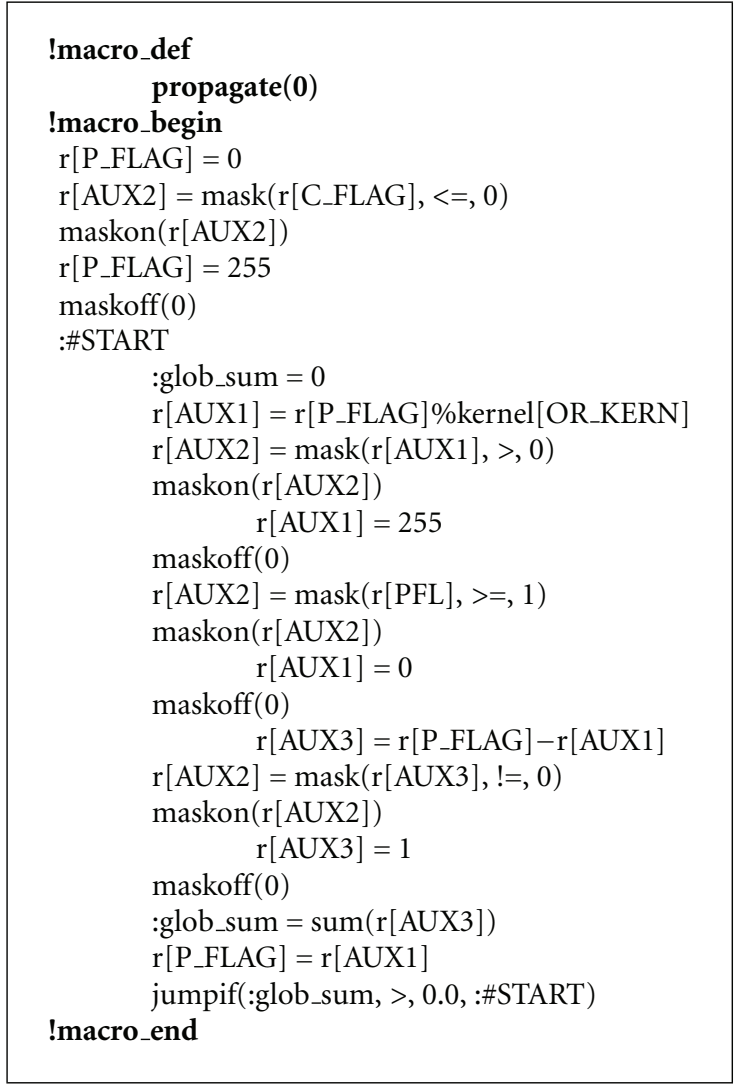

APRON-SCRIPT MACRO

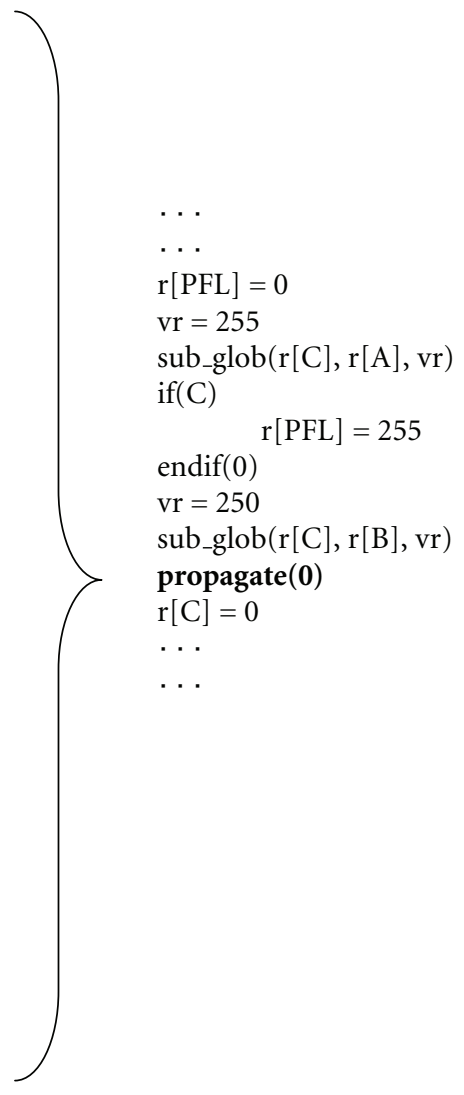

ASPA-SCRIPT ALGORITHM

FIGURE 6: APRON Macro definition of the ASPA function "propagate()". On the right is a snippet of ASPA-Script assembled from macros. On the left is an expansion of the propagate macro written in APRON-Script.

TABLE 1: Descriptions of the benchmark tests performed.

\begin{tabular}{ll}
\hline Test & Description \\
\hline ADD & $\begin{array}{l}\text { Two registers were filled with random values. The } \\
\text { two registers were added and written to a third } \\
\text { separate register } 10000 \text { times. }\end{array}$ \\
MULT & Same as ADD, but with a multiply operation. \\
CONDMULT & $\begin{array}{l}\text { Same as MULT, but the two registers were } \\
\text { multiplied, only where the contents of register } 1 \\
\text { are greater than } \theta \text {, using a mask register. }\end{array}$ \\
& $\begin{array}{l}\text { One register was filled with random values. A } \\
3 \times 3 \text { Gaussian Blur Kernel was used to filter the } \\
\text { register and the result was written to a second } \\
\text { separate register, repeated } 10000 \text { times. }\end{array}$ \\
\hline
\end{tabular}

As an example, Figure 6 shows an implementation of the ASPA "propagate()" function, which has been implemented in APRON-Script. Even though this is one instruction on an ASPA device, it needs several looped APRON operations. However, when the instruction is simulated, it will behave as a single step. This process is transparent to the user and often fast enough due to the efficient operations provided by the APRON core.

The ASPA ruleset is a portable definition of the ASPA device, with which other developers can rapidly create algorithms for the chip, without having the hardware to test them on. This has been achieved without needing to build a custom simulator for the ASPA device. The plug-in feature of APRON can be exploited for additional functionality, including being used as an interface to an actual ASPA device. This makes APRON a complete design, test, and development suite for the ASPA. At present a hardware interface has been implemented successfully for the SCAMP device, where frames of preprocessed data from the SCAMP device can be operated on further by efficient APRON routines.

\section{Benchmarking}

Throughout the development of APRON, a great effort has been made into optimizing the APRON Core, to make it as fast as possible. In order to assess its performance, a set of benchmarks were compiled that measure the execution time of commonly used operations and the performance of APRON, MATLAB (7.01), and unoptimised high-level 

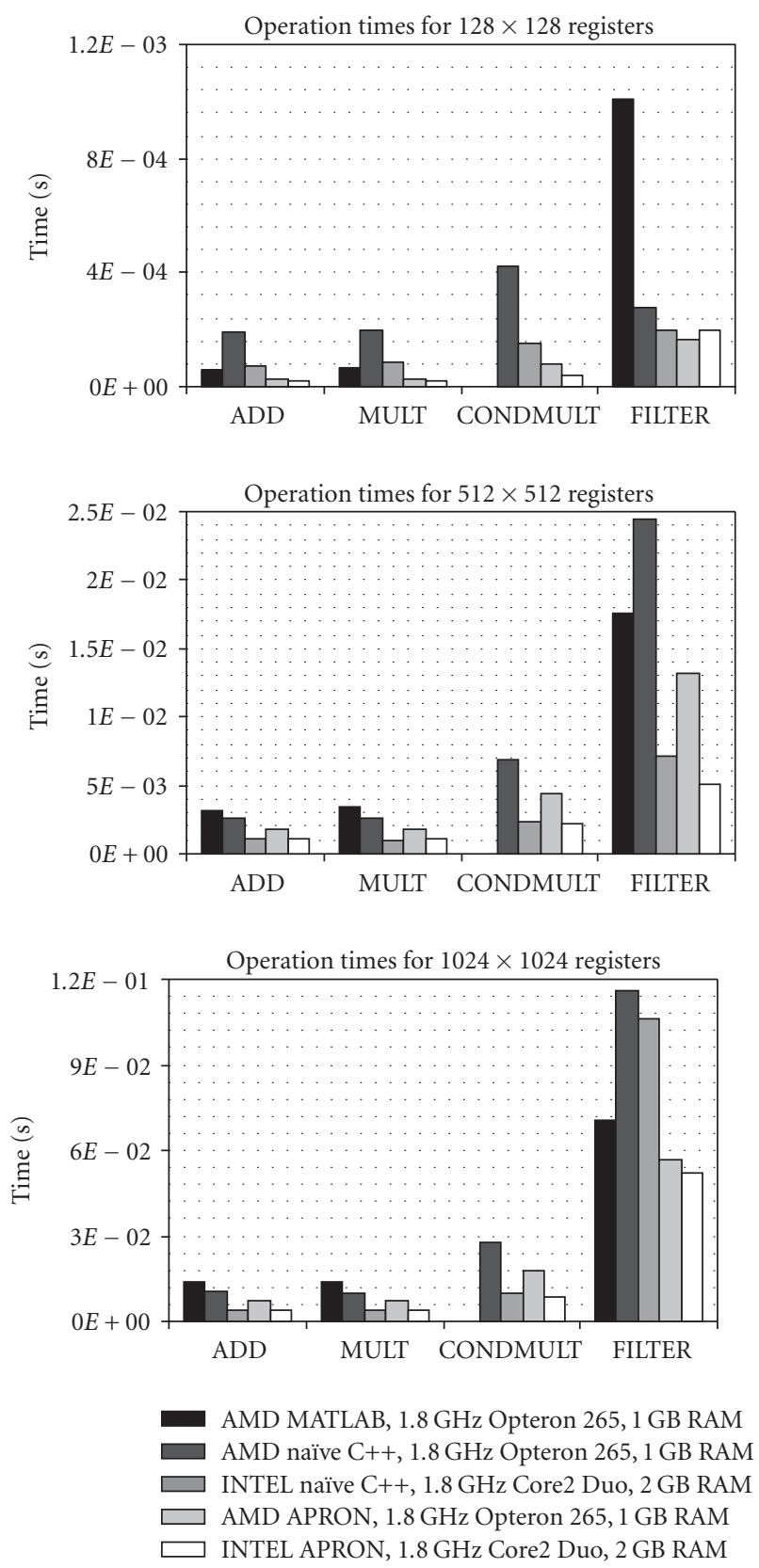

FIGURE 7: Benchmarks showing the performance of different array processing operations on different software platforms.

(naïve) $\mathrm{C}++$ is compared. The tests performed are described in Table 1, and the results are shown in Figure 7.

Two test machines were used with similar specifications, a 1.8 GHz AMD Opteron 265, running Microsoft Windows XP, and a $1.8 \mathrm{GHz}$ Intel Core2 Duo running in Windows Vista. It was not possible to benchmark MATLAB programs on the Intel platform at this time. The benchmark results show that APRON is faster than MATLAB at all tasks, often by a factor of 3 , and faster than a naïve $\mathrm{C}++$ approach in almost all cases. The results have shown interesting differences between Intel and AMD processors, with AMD being on the whole slower. This is most likely due to the nature of AMD's implementation of Intel's SSE technology. The operating system's memory/process management system also contributes to the performance, and this can be seen in the results of the larger register filtering tests, as SSE is not used for this operation.

APRON performs $128 \times 128$ array operations using single precision floats in tens of microseconds, which is suitable for a great range of experiments and applications. Execution times can largely be reduced four-fold if the change to using 8 -bit integers is made.

\section{Conclusion}

This paper introduced an efficient software package used for the simulation and prototyping of cellular processor arrays. This software is useful throughout the life cycle of a cellular processor array-based device, from the initial design, modelling, and prototyping of the hardware, through to being used as an algorithm development platform, a simulator, and even a hardware interface. An example application of modelling a hardware CPA was presented, and the benefits that APRON provided to the user experience of working with the CPA were highlighted. APRON software proves to be faster than other software (and some hardware) environments for the tasks of emulating, prototyping, and simulating cellular processor arrays and can even be used as a stand-alone "array processing" system in many applications. Software tools like APRON can encourage the "massivelyparallel" programming style and provide tools that ease the transition from software to massively parallel hardware implementations.

It is interesting to see that "virtual" massively-parallel devices created in software are beginning to compete with custom ICs and FPGAs in terms of computational performance, at comparatively lower cost. With commercial CPU manufacturers producing more parallel, lower power devices, perhaps software environments such as APRON will become more appealing to researchers interested in finegrain processor arrays and their applications in areas such as computational neuroscience and image processing.

\section{Acknowledgment}

This work has been supported by the EPSRC, Grant no. EP/C516303.

\section{References}

[1] K. E. Batcher, "Design of a massively parallel processor," IEEE Transactions on Computers, vol. 29, no. 9, pp. 836-840, 1980.

[2] S. F. Reddaway, "The DAP approach," in Infotech State of the Art Report on Supercomputers, vol. 2, pp. 309-329, Infotech International, Maidenhead, UK, 1979.

[3] W. D. Hillis, The Connection Machine, MIT Press, Cambridge, Mass, USA, 1985.

[4] Y. I. Fet, Parallel Processing in Cellular Arrays, John Wiley \& Sons, New York, NY, USA, 1995.

[5] A. Lopich and P. Dudek, "Global operations on SIMD cellular processor arrays: towards functional asynchronism," in Proceedings of the International Workshop on Computer Architectures for Machine Perception and Sensing (CAMPS '06), pp. 18-23, Montreal, Canada, September 2006. 
[6] P. Dudek, "Implementation of SIMD vision chip with $128 \times$ 128 array of analogue processing elements," in Proceedings of IEEE International Symposium on Circuits and Systems (ISCAS '05), vol. 6, pp. 5806-5809, Kobe, Japan, May 2005.

[7] A. Zarandy, M. Foldesy, P. Szolgay, S. Tokes, C. Rekeczky, and T. Roska, "Various implementations of topographic, sensory, cellular wave computers," in Proceedings of IEEE International Symposium on Circuits and Systems (ISCAS '05), vol. 6, pp. 5802-5805, Kobe, Japan, May 2005.

[8] A. Rodríguez-Vázquez, G. Liñán-Cembrano, L. Carranza, et al., "ACE16k: the third generation of mixed-signal SIMDCNN ACE chips toward VSoCs," IEEE Transactions on Circuits and Systems I, vol. 51, no. 5, pp. 851-863, 2004.

[9] M. Laiho, J. Poikonen, P. Virta, and A. Paasio, "A $64 \times$ 64 cell mixed-mode array processor prototyping system," in Proceedings of the 11th IEEE International Workshop on Cellular Neural Networks and Their Applications (CNNA '08), Santiago de Compostela, Spain, July 2008.

[10] J. A. Kahle, M. N. Day, H. P. Hofstee, C. R. Johns, T. R. Maeurer, and D. Shippy, "Introduction to the cell multiprocessor," IBM Journal of Research and Development, vol. 49, no. 4-5, pp. 589-604, 2005.

[11] D. Wentzlaff, P. Griffin, H. Hoffmann, et al., "On-chip interconnection architecture of the tile processor," IEEE Micro, vol. 27, no. 5, pp. 15-31, 2007.

[12] L. Seiler, D. Carmean, E. Sprangle, et al., "Larrabee: a many-core $\times 86$ architecture for visual computing," $A C M$ Transactions on Graphics, vol. 27, no. 3, article 18, pp. 1-15, 2008.

[13] W. J. Dally, U. J. Kapasi, B. Khailany, J. H. Ahn, and A. Das, "Stream processors: progammability and efficiency," ACM Queue, vol. 2, no. 1, pp. 52-62, 2004.

[14] D. Balya and T. Roska, "Face and eye detection by CNN algorithms," Journal of VLSI Signal Processing Systems for Signal, Image, and Video Technology, vol. 23, no. 2-3, pp. 497$511,1999$.

[15] B. E. Shi, "An eight layer cellular neural network for spatiotemporal image filtering," International Journal of Circuit Theory and Applications, vol. 34, no. 1, pp. 141-164, 2006.

[16] J. Sirosh and R. Miikkulainen, "A unified neural network model for the self-organization of topographic receptive fields and lateral interaction," in Proceedings of the Joint Conference on Information Sciences (JCIS '94), pp. 282-285, Durham, NC, USA, November 1994.

[17] D. Balya and B. Roska, "Cellular wave computing the multi-channel mammalian retina model by the Bi-I cameracomputer," in Proceedings of the 10th IEEE International Workshop on Cellular Neural Networks and Their Applications (CNNA '06), pp. 1-12, Istanbul, Turkey, August 2006.

[18] D. R. W. Barr and P. Dudek, "A cellular processor array simulation and hardware prototyping tool," in Proceedings of the 11th IEEE International Workshop on Cellular Neural Networks and Their Applications (CNNA '08), pp. 213-218, Santiago de Compostela, Spain, July 2008.

[19] Intel, AP-809, "Real and complex FIR filter using streaming SIMD extensions," version 2.1, January 1999.

[20] D. R. W. Barr, S. J. Carey, A. Lopich, and P. Dudek, "A control system for a cellular processor array," in Proceedings of the 10th IEEE International Workshop on Cellular Neural Networks and Their Applications (CNNA '06), pp. 176-181, Istanbul, Turkey, August 2006.

[21] B. Cope, P. Y. K. Cheung, W. Luk, and S. Witt, "Have GPUs made FPGAs redundant in the field of video processing?" in Proceedings of IEEE International Conference on FieldProgrammable Technology (FPT '05), pp. 111-118, Singapore, December 2005.

[22] L. O. Chua and L. Yang, "Cellular neural networks: applications," IEEE transactions on circuits and systems, vol. 35, no. 10, pp. 1273-1290, 1988.

[23] J. A. Bednar, "Understanding neural maps with topographica," Brains, Minds, and Media, vol. 3, Article ID bmm1402, 2008.

[24] D. R. W. Barr, P. Dudek, J. M. Chambers, and K. Gurney, "Implementation of multi-layer leaky integrator networks on a cellular processor array," in Proceedings of IEEE International Joint Conference on Neural Networks (IJCNN '07), pp. 15601565, Orlando, Fla, USA, August 2007.

[25] E. M. Izhikevich, "Simple model of spiking neurons," IEEE Transactions on Neural Networks, vol. 14, no. 6, pp. 1569-1572, 2003.

[26] P. Dudek, "Accuracy and efficiency of grey-level image filtering on VLSI cellular processor arrays," in Proceedings of IEEE International Workshop on Cellular Neural Networks and Their Applications (CNNA '04), pp. 123-128, Budapest, Hungary, July 2004. 